\title{
Movimientos de población en la frontera a través de una familia
}

\section{de inmigrantes}

\author{
Os movimentos de população na fronteira por uma família de imigrantes
}

Population movements at the border by a family of immigrants

\author{
Cecilia Chagas; Eugenia Liendo; Marcia Peña; Nicolás López; Victoria Manzoni ${ }^{1}$
}

\begin{abstract}
Resumen
A lo largo de la historia, se han producido diversos fenómenos que impulsaron a las personas a trasladarse de un lugar a otro, llevando consigo costumbres, tradiciones, ideología y muchos otros aspectos de su vida con los cuales intentan preservar su identidad a través del tiempo. Este trabajo se enmarca dentro de una de las etapas de ejecución del Equipo de Investigación en Estudios de Frontera EMEFRO, que está siendo realizado por un cuerpo de docentes del Centro Regional de Profesores del Norte, en conjunto con el Museo del Patrimonio Regional. En esta instancia, se ha tomado como unidad de análisis a una familia de inmigrantes alemanes de apellido Fros, provenientes del este de Brasil y radicados en la región norte de la Banda Oriental en el Siglo XIX, hoy Uruguay. El objetivo central de este trabajo, es el estudio de las consecuencias de los movimientos de población a través de la frontera, mediante la indagación de aspectos materiales e inmateriales reflejados en dicha familia. Se busca identificar y comprender elementos identitarios que aún se conservan o no, de las raíces europeas de los Fros.
\end{abstract}

Palabras clave: Migración; frontera; cultura; identidades.

\section{Resumo}

Ao longo da história, tem havido vários eventos que levaram às pessoas a se deslocarem de um lugar para outro, levando costumes, tradições, ideologia e muitos outros aspectos da vida com os quais eles tentam preservar a sua identidade ao longo do tempo. Este trabalho faz parte de uma das fases de implementação da equipe de pesquisa em Estudos de Fronteiras, que está sendo conduzido por um grupo de professores do "Centro Regional de Professores do Norte", em conjunto com o Museu departamental de Rivera. Neste caso, a unidade de análise é uma familia de imigrantes alemães de sobrenome Fros, do leste do Brasil e com base na região norte da Banda Oriental no século XIX, hoje Uruguai. O principal objetivo deste trabalho é o estudo das consequências dos movimentos de populações através da fronteira, por meio da investigação de aspectos tangiveis e intangíveis refletidas nesta família. Pretende-se identificar e compreender elementos de identidade que ainda permanecem ou não nas raizes europeias dos Fros. Investigar sobre esta questão é um mecanismo que permitirá novas perspectivas sobre a região, sobre a cultura e a sociedade fronteiriça, permitindo que adquira maior importância e interesse a nivel regional e internacional.

Palavras-chave: Migração; fronteiras; cultura; identidade.

Abstract

Throughout history, there have been several events that prompted people to move from one place to another, carrying customs, traditions, ideology and many other aspects of life with which they try to preserve their identity over time. This work is part of one of the stages of implementation of the Research Team EMEFRO Border Studies, which is being conducted by a body of Regional Center of Northern Teachers (CeRP), in conjunction with the Regional Heritage Museum. In this instance, it has been taken as the unit of analysis to a family of German immigrants surnamed Fros, from eastern Brazil and based in the northern region of the Eastern Band in the nineteenth century, today Uruguay. The main objective of this work is the study of the consequences of population movements across the border, through inquiry of tangible and intangible aspects

\footnotetext{
${ }^{1}$ Centro Regional de Profesores del Norte; Rivera, Departamento de Rivera, Uruguay; ceci.maca@hotmail.com.
} 
reflected in this family. It seeks to identify and understand identity elements that still remain or not, European roots Fros.

Keywords: migration; border; culture; identity.

\section{Introducción}

El siguiente trabajo se estructurará de la siguiente manera, en primer lugar se abordarán los diferentes conceptos (cultura, identidad, frontera, matrimonio, familia, migración, inmigración, memoria colectiva, ideología, sociedad y eurocentrismo), utilizados para enfocar al tema principal, los movimientos en frontera. A partir de la conceptuación se estará brindando un breve contexto tanto de América como de Europa y principalmente de Alemania. La cronología que se tiene en cuenta con las últimas décadas del siglo XVIII y principios del siglo XIX. El objetivo principal de esta contextualización es llegar a comprobar las hipótesis y objetivos que se tienen. Por último se estará realizando un análisis de los diferentes hallazgos que se tiene, fuentes primarias, fotografias, bautismos, propiedades y entrevistas, y fuentes secundarias bibliografía. Estos son de gran relevancia a la hora de la conclusión, ya que su utilidad es contrastar estos materiales .

\section{Estado del arte:}

Se le llama sociedad a aquel conjunto de personas que tienen un fin en común, que mediante diferentes mecanismos logran convivir en un mismo territorio, es por esto que Giddens (1999) sostiene que existen reglas, leyes, jerarquía, las cuales no son las mismas, sino que cada sociedad se caracteriza por una organización diferente. Por ende Castillo (2012) menciona que los seres humanos no sólo viven juntos, sino que se encuentran interactuando continuamente, respondiendo unos a otros, orientando sus acciones en relación con la conducta de los demás y siempre con un doble fin: allegarse a algo que necesitan y ayudar a otros a conseguirlo. Además aprende sus modos habituales de comportamiento, las normas y valores aceptados, la cultura, costumbres, el lenguaje, la ideología, entre otros aspectos que incluye la vida en sociedad.

Por lo tanto los diferentes grupos de individuos transitan procesos de interacción social fundados en variadas formas de comunicación, intensos y duraderos en el tiempo, con intereses en común, ya sean instrumentales, afectivos, morales derivados de rasgos comunes, entre muchos otros, Bohannan, (1992). Uno de los diversos grupos sociales en los que interactúan los individuos, es el de la familia, la cuna de la socialización primaria.

Por lo tanto la familia forma parte de la sociedad y se puede decir que está compuesta por dos o más miembros, aunque los conceptos que se han de utilizar hoy son muy 
diferentes a los que se utilizaban en otros tiempos, Castillo (2012). Como todo cambia y evoluciona, la familia es uno de ellos. Cabe agregar aquí que Ackerman (1988) sostiene que ninguna persona vive su vida en soledad, comparte los primeros años de vida, casi exclusivamente con los miembros de su familia, la cual puede definirse como la unidad básica de desarrollo y experiencia, de realización y fracaso, siendo también la unidad básica de la enfermedad y la salud. Además la familia toma para sí, de la sociedad, emociones, pensamientos y juicios, al mismo tiempo que le proporciona a esta, emociones y puntos de vista propios.

Por otro lado se abarca conceptualizaciones que son necesarias para el desarrollo de la misma investigación, por ende se tuvo que abarcar conceptos como migración, inmigración, frontera, identidad, cultura, ideología y memoria colectiva. En cuanto al concepto de migración, se tuvo en cuenta la visión de Antonio Aja Díaz, el cual en una de sus publicaciones menciona los movimientos poblacionales, estos son de carácter internacional como las migraciones que son producto de "un cambio global y cambios posteriores" (AJA DÍAZ, 2004, Pág.1). Posiblemente devengan de temáticas múltiples, pero seguramente estos movimientos poblacionales producen impactos en las relaciones internacionales y diversifican la riqueza étnica y cultural modificando los conceptos de fronteras. Como causante de las migraciones este autor nos da una visión enfocada a las desigualdades económicas, aunque sin dejar de nombrar otros motivos como tendencias demográficas, insuficiencias estructurales del desarrollo y falta de oportunidades laborales. A Su vez nos da un panorama del escenario de los flujos migratorios resaltando los flujos financieros. Las oleadas migratorias siempre existieron, desde tiempos remotos. Es un común denominador en la historia de la humanidad. “La migración complementó la expansión del comercio” (AJA DÍAZ, 2004, pág. 2) y a su vez contribuyó a la creación de las naciones y territorios, provocó modificaciones en la sociedad dejando connotaciones culturales.Las ciudades recibieron estas oleadas migratorias y alimentaron su crecimiento y desarrollo. Según el autor en ese periodo temporal el mundo conoció dos grandes oleadas migratorias, las europeas y las asiáticas.

Otro concepto que va muy de la mano con el de migración es el de inmigrante, que es aquel que se traslada de un lugar a otro por diferentes motivos, ya sea económico, como sociales y políticos. Inmigran a otros lugares en busca de una mejor calidad de vida,"E1 inmigrante es un sujeto construido socialmente y fragmentado institucionalmente (...) portador de nuevas culturas" (LOBERA PÉREZ-MÍNGUEZ, pág. 12). Si bien los conceptos de Migración e inmigración son importantes la definición de frontera es aún de más relevancia por la realidad en que encontramos inmersos "Los niveles de permeabilidad de las 
fronteras pueden ser diversos: fronteras en que los límites los han puesto los Estados, totalmente artificiales; pero también las fronteras que los propios pueblos imponen." (MARÍA DEL ROSARIO BOTTINO, 2009, Pág: 4). A partir de la concepción historiográficahistórica, se puede decir que es un espacio histórico o territorial que se conforma por una multiplicidades de elementos ya que se encuentra dentro de un marco de acontecimientos sociales del pasado.

Es por esto que "cada una constituida a partir de la combinación de las categorías espaciales (zona, región, territorio), fenómenos y procesos que ocurren en los espacios de frontera y los sujetos sociales involucrados en tales procesos" (ARRIAGA RODRÍGUEZ, 2008, pág 73). Además cabe agregar que según Bottino (2008) es la terminación de lo que se llama unidad político-territorial que se extiende un territorio y comienza otro, en otras palabras y sumándole el pensamiento de Turner (1893), él sostenía que la frontera trascurrió por algunos procesos, en primer lugar la frontera del cazador, la del minero, la del agricultor y por último la urbana, con la creación de las ciudades.

Existen elementos, costumbres, cultura, Quijano (2014) refiere a patrones históricamente invariables, tan íntimos en una sociedad, que se conservan, en el tiempo y también se puede trasladar en el espacio, sin ser alterados estos patrones como refiere el autor. Las ideología, la cultura y sus aspectos son conservados a través del tiempo en la memoria colectiva, aquello que la colectividad quiere conservar"(...) no retiene del pasado sino lo que todavía está vivo o es capaz de permanecer vivo en la conciencia del grupo que la mantiene"(MAURICE HALBWACHS, Pág: 127), al hablar de colectividad como grupo de personas tomamos como ejemplo la familia. Sostiene el autor que no existe una memoria individual, nuestra memoria se va estructurando desde que nacemos con lo social, según el grupo a que pertenezcamos. “(...) nociones que nos dominan más que otras, con personas, grupos lugares, fechas, palabras y formas de lenguaje (...) con la vida material y moral de las sociedades que hemos formado parte" (MAURICE HALBWACHS; En Johan Méndez-Reyes, Pág.: 126). Con estos elementos que describe el autor, se pueden identificar en los hallazgos, entrevistas, fotos, y el análisis mismo de los aspectos de la cultura, cuales aspectos quisieron preservar, cuales conservan en la memoria colectiva.

\subsection{Técnicas y métodos:}

Para comenzar este trabajo se definió la metodología a seguir, en esta oportunidad es la metodología inductiva porque partimos de un presupuesto para alcanzar una conclusión, ya que se analiza de lo particular partiendo de observaciones, a lo general con el análisis de 
documentos. El enfoque que se utiliza para esta investigación es de corte cualitativo y antropológico histórico. Para la realización de la misma, se manejan diferentes estrategias, como la observación, el análisis de documentos, y entrevistas en profundidad a diferentes actores. Las entrevistas se realizaron a miembros de la familia Fros y a otras personas que aportaron información relevante para el desarrollo del trabajo; las observaciones son en las estancias pertenecientes a dicha familia y el análisis es de documentos relacionados con esta familia, todo se realizó siempre con el permiso de las personas.

Luego de realizar la búsqueda de documentos, se recurrió a los entrevistados para nuevamente recabar información, se ha encontrado por medio de uno de los entrevistados, quien está realizando una investigación informal, el árbol genealógico de la familia Fros. Este árbol contiene la información necesaria, ya que nos comprueba quien fue el primer Fros en llegar a la región y como fue dejando su herencia.

\section{Hallazgos:}

Se cree pertinente comenzar con una breve descripción del contexto de Brasil y Europa, el porqué de los sentidos de las inmigraciones, las oleadas migratorias, dónde se establecieron, y principalmente permite visualizar algunos elementos de la cultura que conservaron los alemanes Tales aspectos permiten asociar a la cultura de la familia Fros que se analiza en el presente trabajo

El contexto económico y social en el período indicado se destaca principalmente por la plantación del café, en el momento por las grandes dimensiones de las plantaciones, la actividad requería una cantidad de mano de obra. "A lavoura do café constitui um marco na evolução económica do Brasil, transformando o processo de produção. Nascem fazendas com certas características de empresa" (MARLI; CRUXEN; 1976 Pág. 17).

Como riquezas de este territorio estaba la explotación del oro y otros tipos de plantaciones que eran muy productivas, el espacio más codiciado por la fertilidad de sus tierras era la región del Rio Grande do Sul. "Seus campos admiráveis, servidos por um sistema fluvial que constituía inapreciável tesouro, povoados de milhares de cabeças de gado, magnífica herança que nos fora legada pelos primeiros estabelecimentos jesuíticos, e a terra fértil (...)" (AURELIO PORTO, 1996, Pág:7). En lo social y político refiere este autor, que se estaba dando el desarrollo de las haciendas, latifundios, los cuales trabajaban en sus grandes extensiones de plantaciones, con mano de obra esclava. En un principio funcionó este sistema, luego con el proceso abolicionista de la esclavitud, esta medida comenzó a no rendir en la productividad, lo que convino a esta política y economía fue invertir en mano de obra libre, 
"Interesse que havia por parte do governo e da classe dominante da colonia em atrair ondas imigratórias, reconhecendo no europeu um elemento mais apto do que u esclavo para ocuparse de atividade agrícolas" (MARLI; CRUXEN,1976, Pág: 16), para esto implantan una política que beneficiaba y atraía a familias europeas para que comenzaran a asentarse en las colonias del Rio Grande do Sul. La preferencia por familias europeas, se da principalmente por su mentalidad de acumulación de riquezas, diferencia que se da con respecto a la mano de obra eslava, un extranjero al ser remunerado, rendiría más en su trabajo. Refieren estos autores que el objetivo de los grandes latifundistas era que estas familias solamente hicieran parte del sistema planificado como mano de obra, y que no adquirirían tierras en el territorio para luego ser propietarios haciendo competencia a sus poderes. Aurelio Porto (1996) Dentro de la preferencia por los extranjeros también hubo la preferencia por algunos especiales, que resultaban elementales, los italianos y alemanes, estos eran famosos por ser muy buenos agricultores, y otro factor bien visto por los portugueses fueron sus técnicas agrícolas, estas llevarían a un desarrollo más rápido de las plantaciones, ya que eran más avanzadas que las que ya utilizaban.

Como ya se ha descrito la fertilidad y virtudes del territorio del Rio Grande do Sul, lugar donde se desarrollan las colonias alemanas en el proceso de la política inmigratoria, pero también sostiene Marli, Cruxen (1976) que se establecieron europeos en Sao Paulo, los cuales eran financiados por hacendados que tenían las grandes plantaciones de café a diferencia de los que se establecieron en Rio Grande do Sul que fueron financiados por el gobierno. La política inmigratoria consistía en la promesa de varios beneficios, dentro de ello también se prometía la nacionalidad. Pero Aurelio Porto (1996) mantiene que el territorio bajo el poder portugués en América era más allá de estos lugares referido, se extendía desde Colonia del Sacramento a Laguna, dos poblaciones que aislaban a Rio Grande del Sul, y en su discurso se puede identificar que durante estas corrientes inmigratorias, y luego con la organización interna del gobierno con respecto a las colonias se dan constantemente movimiento por estas regiones.

Anterior a la mencionada por los autores como política inmigratoria, no existía ningún programa que organizara o amparara a los emigrantes europeos, no se tenía la necesidad que se da en los períodos siguientes, por las plantaciones, pero esto no quiere decir que no existió inmigración, se da pero de una forma discontinua y no eran financiados sus viajes. "não tendo sido orientado por uma política oficial planejada, apresenta-se como um movimento descontinuo em seu direcionamento e intensidade caracterizando-se por momentos de grande afluxo, seguidos de interrupções." (MARLI; CRUXEN, 1976, Pág: 27) 
estas corrientes de inmigraciones se dan a consecuencia de algunos hecho, europeos o del territorio portugués, por esto, los autores plantean algunos acontecimientos que influyeron o no en las decisiones de inmigrar, destacan el período de 1808 en el cual se da la apertura de puertos y luego se les asegura a los inmigrantes propiedades de territorios, se rompe el monopolio portugués del comercio, generando a la vez interés por parte de otros pueblos.

Cuando surge la necesidad de mano de obra libre, y se implanta la política de inmigración comienzan los flujos inmigratorios homogéneos, continuos. "A colonização estrangeira, propriamente dia, começa, para o Brasil, em 1818, com a tentativa de Nova Friburgo(...)Mais tarde, antes da fundação de S. Leopoldo, recebeu também Friburgo um forte contingente de colonos alemães, mandados vir pelo governo imperial(...)" (AURELIO PORTO ,1996, Pág:36-37). Las inmigraciones de alemanes fueron muy irregulares, el objetivo de la política era arraigar a estos inmigrantes a la tierra y que produjeran para desarrollar y abastecer el mercado interno. "uma política deliberada de criar núcleos coloniais voltados para a ocupação e fixação na terra" (MARLI; CRUXEN,1976 Pág. 33). Para lograr el establecimiento de estos inmigrantes a la tierra también era necesario generar patriotismo, sentido de pertenencia del nuevo territorio.

(...) sem intercâmbios de qualquer espécie intelectual e moral, e mandámos que trabalhasse, exigindo-lhe somente fossem ótimo agricultor. E depois, quisemos a força impor preceitos patrióticos, que somente um largo trabalho de fraternidade e amor poderia semear em suas almas. (AURELIO PORTO, 1996, Pág: 223).

En este período de colonización surgen factores que dan lugar a la movilidad social dentro del territorio que estaba en poder de los portugueses, estos factores fueron los traslados de población de unas colonias a otras, "para a cultura do cáñamo, e desejando aproveitar essas terras, resolveu o governo encaminhar para o soul un forte contingente de colonos alemães, cujos resultados positivos se estavam assinalando em Nova Friburgo." (AURELIO PORTO; 1996, Pág.: 37), otro factor que pudo dar lugar a esta movilidad de población fue la desconfianza en algunas autoridades de la política inmigratoria, prometían beneficios y estos no eran cumplidos, "E, para a obtenga de colonizadores, agira de modo a nao recomendar o seu nome. (...) justificar a desconfiança que envolvera a ação de Schaeffer, como se verificados dos contratos firmados em 1824 com os primeiros(...)Ao chegarem ao Rio de Janeiro, souberam eles que eram falsas as promessas de Schaeffer" (AURELIO PORTO, 1996, Pág:38-39). Otro factor a destacar fue la distribución de tierras a los inmigrantes, al no ser equitativas, no se medían los lotes de tierras, generándose así problemas. 
En este contexto de movilidad, no se afirma pero sí se muestra la posibilidad de que allí sea el momento donde la familia Fros, inmigrantes alemanes, luego de adquirir nacionalidad brasilera se dispersara por las tierras dominadas por los portugueses y se estableciera en la región norte de la Banda Oriental, donde hoy es Laureles, departamento de Tacuarembó-Uruguay. Región que en el momento no existía ningún tipo de límite, y donde en un sentido amplio esta familia se apoderó del territorio que gustó en su extensión y dónde allí se estableció y creo su establecimiento para vivir con su familia "(...) el padre de él contaba y el abuelo, que en la época del ganado silvestre y que salió un decreto del gobierno que bueno, todo aquel que cercara su predio y lograra sustentar grandes cantidad de animal el consideraba propietario, seguramente él era medio baqueano en el tema y le hicieron cercos de pierda(...)" (Entrevistado: D. F).

La economía de las colonias alemanas en Brasil se desarrolló principalmente en la agricultura, el producto de gran interés fue el trigo, esta fue tan importante para sus abastecimientos como para el comercio, favoreció al desarrollo del poblamiento de la colonias "(...) mandando ao Rio Grande duas embarcações" para transportar o trigo alí adquirido. (...) foram grandes remessas de trigo e farinha para o reino." (AURELIO PORTO, 1996, Pág.: 9). Pero su gran auge se desarrolla hasta que se da una peste en las plantaciones. Pero no sólo este tipo de plantación se desarrolló sino que el autor AURELIO PORTO (1996) describe la variedad económica que desarrollaban en las colonias. Además del trigo, el tabaco, la mandioca, caña de azúcar, algodón, castañas, batatas, cacao, moras y además desarrollaron el engorde de porcinos.

Tales se las puede comparar con las actividades económicas que por los relatos orales, entrevistas, a personas de apellido Fros cuentan lo que de generación en generación ha perdurado, y se puede apreciar que tienen muchas similitudes. En los siguientes relatos se puede apreciar como desarrollaban el trigo, tenían una economía autosuficiente, y la actividad ganadera también.

(...) que hacían bombones de fruta, de la fruta que se cultivaba allí, eran muy muy por la agricultura, este, plantaban árboles frutales, hacían orejón, por ejemplo cuando en la época del durazno, dando heladas y cosas, todo eso iban guardando para los acontecimientos."(Entrevista: T.R.S) "(...)principalmente criaban ganado, digo era más bien ganadero los campos, también plantaban, obviamente, pero más bien se plantaba, la agricultura era para el consumo de la propia familia, porque eran familias muy numerosas,(...)" (Entrevista: A.F) "En casa se producía el trigo molido a mano, se hacia la mazamorra, la fruta que se comía... se comía la que había allí(...) la alimentación se hacía todo ahí, incluso se hacía la harina(...)"(Entrevista: A.F)"(...)aquí se comía el durazno, la naranja, el membrillo, el limón, todo era producido así, y las carneadas era una cosa tradicional, se carneaba vaca y todo eso y... bueno llegado el invierno se tenía la costumbre de engordar un chancho grande, 
hasta el punto de no levantarse más, se lo encerraba y se le daba comida, comida, comida, entonces ya el animal no se levantaba más, entonces ahí cuando no se levanta esperaban una semana y a la semana lo carneaba, entonces se carneaba una vaca y al chancho, y ahí se le sacaba el tocino, morcilla, charle, se hacían los embutidos. (Entrevista:A.F)"(...)molíamos el maíz, porque había que quebrar el maíz para darle a los pollitos, se criaban gallinas y eso, era todo un... y era tradicional en casa carnear una vaca todos los meses, todos los meses... por la cantidad de gente y otra cosa que desapareció es el ganado criollo y las vacas criollas (...) porque en casa habían animales criollos que eran los que se carneaban más, la carne más durita, ya no existe más, las ovejas criollas eran muy preciadas, su lana que justamente se utilizaba, porque en casa se le quitaba la lana a la oveja y se hacían los colchones, frazadas, muchas cosas, todo era hecho allí, se lavaba la lana y se hacían las cosas. (Entrevista: A.F)

Desde un punto de vista más específico de lo ocurrido con la cultura de estas poblaciones establecidas en las colonias alemanas en Brasil, se fue atravesando por un proceso por el cual Brasil buscaba establecer como tal su cultura, su nación, un orden en lo cultural, a su vez buscó arraigar al extranjero, en este caso específico al alemán por medio del patriotismo.

\footnotetext{
Nosso verso de procurar em outras civilizações o modelo para tudo; o nosso impatriotismo que prefere um estrangeirismo caricato para vestir ideias genüinamentenossa, com roupagens mirabolantes de exotismo alienígenas, fazendonos caudatários de outros povos; o desconhecimento do caráter e do grão de brasilidade(...) (AURELIO PORTO, 1996, Pág:223).
}

Aurelio Porto (1996), por lo tanto lo que ocurre es que por un lado la cultura brasileña adquiere aspectos de la cultura de estos europeos y los europeos, alemanes adquieren de los brasileños, se va formando a traves de este proceso una fusión entre las culturas. Cuando se habla de cultura se habla de lengua, religión, costumbres, técnicas de construcción, agrícolas, y muchos más. Pero también ocurre que los alemanes como grupo conserven algunos aspectos originarios que los hace únicos, como la fama de trabajadores, buenos agricultores, los describe como ordenados, y muy disciplinados. "(...) aspeto, que caracteriza a índole de um povo, não deslustrou a fama de ordeiros, de que sempre gozaram os alemães. (...)o espírito de ordem, de disciplina da digna colonização alemã e de seus descendentes, no Rio Grande do Sul. O dr. L Truda, em seu trabalho tantas vezes citado, (...)" (AURELO PORTO, 1996, Pág: 224-225). La forma que utilizan para conservar estas características, y la lengua, que es uno de los elementos más importantes para unificar, fue mediante la educación. A pesar de que el gobierno luego utilizara la misma herramienta para unificar con la lengua portuguesa, Aurelio Porto, (1996) promovían el idioma nacional. Este elemento se lo identifica también en la familia, ya que se manejaban con el idioma portugués. 

e-ISSN 2016/Atual: 2525-7870 | e-ISSN 2015/2016: 2447-018X

(...) el idioma, el idioma es decir... que se caracteriza por ejemplo como el portuñol, pero el... este primer Fros por datos él era... tenía un acento bastante, bastante portugués, porque como el vino de Brasil, no digo cerrado del todo digo... y inclusive el padre de papá, por los datos, mi abuelo era bastante, bastante abrasilerado (...) (Entrevista: D.F).

Otra estrategia que adoptan los portugueses para generar el sentido patriótico fue mediante la evangelización."(...) a feição moral, a santidade, desse primeiro evangelizador das selvas rio-grandenses que foi o padre Roque, mártir admirável de Caaró. (...) deixaram traços luminosos e inapagáveis de seu cristianismo esforço, para elevar a cultura do Rio Grande do Sul.” (AURELIO PORTO, 1996, Pág: 227)

Mediante la línea del árbol genealógico brindado por A. F, se pudo iniciar desde el origen de esta familia, ya que no se encuentran fuentes escritas oficiales que comprueben como llegó Guillermo Alberto Fros Texeira, en una primera instancia a la localidad de Blanquillo, Durazno y luego a la de Laureles, Tacuarembó. Igualmente se pudo saber llegar a encontrar información por parte de los relatos familiares que fueron pasando de generación en generación. Además se comprueba la llegada de Guillermo por medio de un documento que fue escrito como un relato familiar y que se conserva en primera estancia la estancia, en Laureles.

A ver, a estos son esto, es letra de mi padre, esto es de papá, sí. Aa mira, mira lo que puso papá acá, mira que bueno, dice así... mira, yo no me había dado cuenta de eso, se ve que vos no dejas nada pa atrás, mira dice: Don Guillermo Alberto Fros nació en Brasil en el año 1801, y después vino al Uruguay y se radicó en el departamento de Durazno, en la localidad de Blanquillo, tomá, en la capilla de Farruco, en esos años se encontraba ahí salió un decreto del gobierno que toda persona que domesticara ganado, mira lo que yo te decía, bagual quedaba con los mismos de propiedad, ta fue así que se formó de un buen rodeo, don Alberto Guillermo Fros estando radicado en las mencionadas localidades del departamento de Durazno, contrajo enlace con doña Ana Mauricia Duarte. En matrimonio nacieron 14 hijos, 9 mujeres y 5 varones, sigue acá, si mira, con lujo y detalle, las mujeres son: Ana Mauricia, aa no, son: María Ilia, Felicia, Amelia, Eludices, Jerónima, Toribia,Juana, Ana Mauricia y Licencia. Los varones, Florentino, él que está ahí en la sala, Florentino Alberto, Toribio, Pedro, José Luis, Juan Franscsco, en el año 1857, el 28 de enero Don Guiilermo Alberto Fros adquirió el campo entre el arroyo cañas y Laureles del río Tacuarembó hasta la cañada llamada, Gruta de las tacuaras, que es ahí cerca, Gruta de las tacuaras, y la que desagua en arroyo cañas, frente a hoy escuela 102, paró a adherir a una población que vivía cerca del llamado Paso Viejo de Laureles allí, después por el año 1865, 1866 construyó donde hoy es el establecimiento y más tarde, exactamente en el año 1869 construyó la otra parte de la casa. 1869, que es aquella parte de allá, en la que ta al correr del galpón, y dice que en la historia este pedazo acá es 6 o 8 años mayor, que fue la primer parte que construyeron, allí, allí nomás esta la era del año, en una piedrita, después saca foto de ella, una piedrita con la era del año, y así se ve que que es otro asunto, a sí es sí, es propiedad de mi abuelo, el campo que adquirió fue adquirido por Guillermo Alberto Fros, tatarabuelo de papá(...)ubicado ente los arroyos Laureles y cañas y el río Tacuarembó, el establecimiento cuenta actualmente con 348 hectáreas y lo explota mi padre, cuya firma Don Daniel y Cristina. 
(Entrevista: D.F)

A partir de parte del árbol genealógico fue necesario recabar documentosBautismos- en la curia de Tacuarembó y con el texto redactado por Dr. Juan Villegas S. J que se encontró en la página web de la parroquia, se puede analizar desde el punto de vista cultural religioso, que esta familia al llegar a la región y mediante el proceso evangelizador pasa a ser católica, ’De la sede montevideana provenía personal evangelizador y las directivas y nombramientos que posibilitaban una mejor atención del Norte" (Dr.JUAN VILLEGAS, Pág:13) pero lo que no se sabe es si esta religión fue adquirida en la región, ya la traían de Brasil o de su país originario, Alemania. Aunque probablemente ya fueran católicos en Brasil antes de llegar al Norte de la Banda Oriental, porque como lo sostiene Villegas, los portugueses también eran Católicos. El Norte del Uruguay siguió sin conocer otras religiones y cultos. Sostiene este autor que los portugueses también eran católicos. Cabe destacar que esta religión es la imperante en Europa, y que probablemente ya la practicaran desde sus orígenes, o sea en Alemania.

Además lo que nos confirman estos documentos es que son de nacionalidad Brasilera y que llegaron a la región por la frontera septentrional de la Banda Oriental, ya que según Juan Villegas ( Pág, 19), en aquel entonces en el norte de esta región era común la penetración brasilera. Otro factor que nos indica la nacionalidad brasilera es el orden de sus apellidos que luego al pasar a nacionalidad uruguaya cambian. En Brasil el primer apellido de una persona es el materno y en segundo lugar el paterno, a la inversa se desarrolla en Uruguay, es decir el primer apellido es el del padre y el segundo de la madre.

Traducción de uno de los bautismos que confirma lo mencionado anteriormente:

\begin{tabular}{|l|l|}
\hline N 912 & $\begin{array}{l}\text { El día quince de Diciembre de mil ochocientos ochenta y uno, el teniente cura Don } \\
\text { Benito Larraga autorizado por el in pancrito cura Vicario de San Fructuoso de }\end{array}$ \\
Contrajo matrimonio el & $\begin{array}{l}\text { Tacuarembó bautizó solemnemente a Fidelis que nació el veintitrés de Abril del año } \\
\text { de } 1944 \text { en esta con }\end{array}$ \\
Rosalina Sotto. Conte & Angélica Morais Brasileros Abuelos paternos Don Gullermo Alberto Fros y Doña Ana \\
P.A Emilio Grenlich & Mauricia Duarte, abuelos maternos Don Manuel Rodríguez de Morais y Doña \\
& Alegandra Rodríguez; fueron padrinos Don Juan Francisco Fros y Doña Martina \\
& Rodríguez a quienes advirtió el parentesco espiritual y demás obligaciones de que \\
& certificó \\
& José M. Semerio Vicario
\end{tabular}

Tableta -1- Fuente: Transcripción de un bautismo recabado en la Curia de Tacuarembó "San Fructuoso" Libro 4, pág. 119 
RELACult - Revista Latino-Americana de Estudos em Cultura e Sociedade

Revista Latinoamericana de Estudios en Cultura y Sociedad | Latin American Journal of Studies in Culture and Society V. 02, Ed. Especial, dezembro, 2016, p. 419-437| periodicos.claec.org e-ISSN 2016/Atual: 2525-7870 | e-ISSN 2015/2016: 2447-018X

El tercer hallazgo fue la comprobación de la localización de la primera estancia construida por la familia analizada, o sea por Guillermo Alberto Fros. Se localizó las dos estancias que se encuentran en la región, la primera que se encuentra en Laureles, Tacuarembó al norte del Uruguay, la cual fue construida en el año 1865 y se encuentra hoy en poder D.F (uno de los entrevistados) y la segunda la cual está en manos de C.F.P que se encuentra en Cañas, Tacuarembó y fue construida por un hijo de Guillermo, Toribio en año 1891, Toribio Alberto, Fros Duarte.

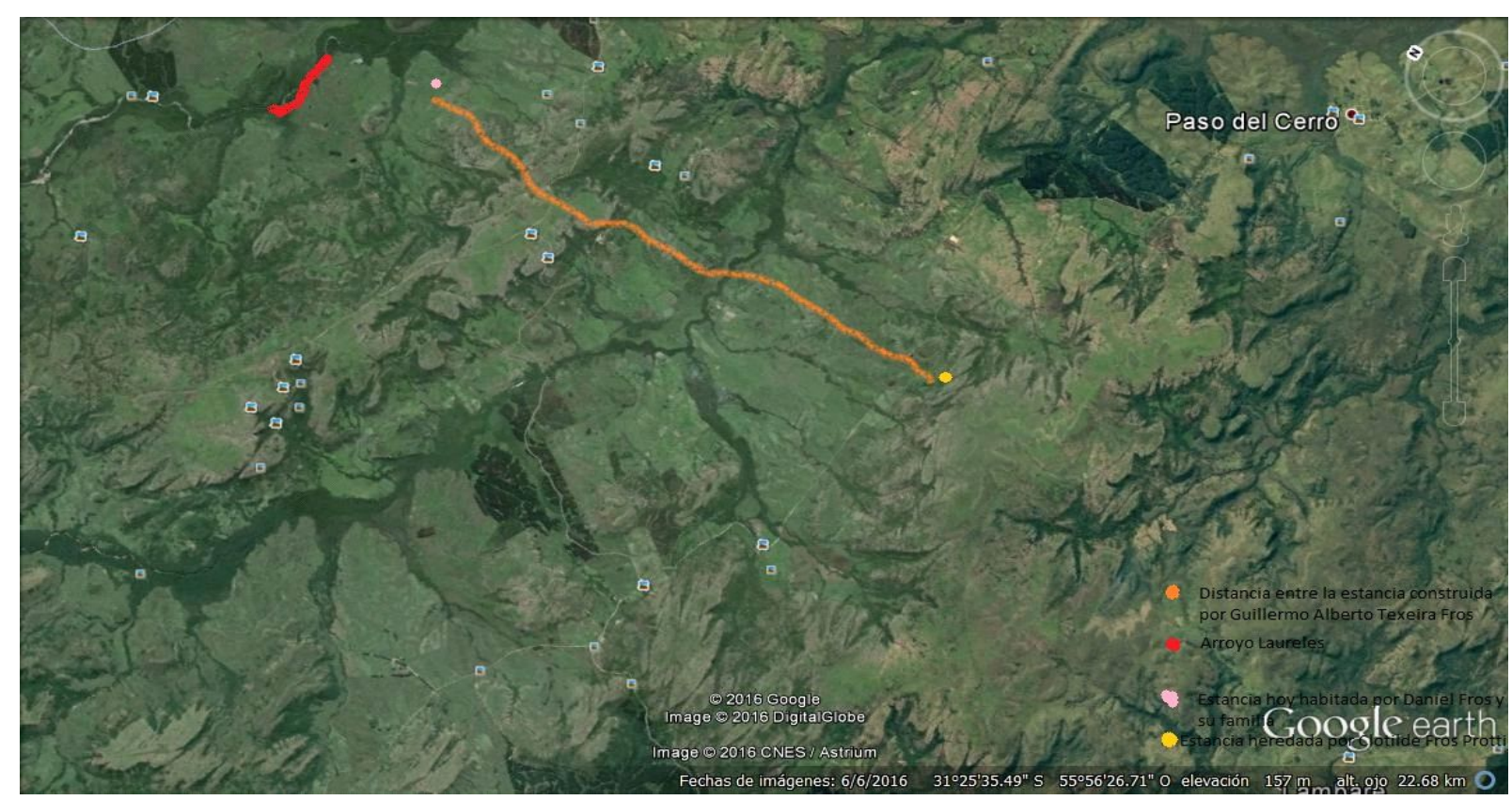

Mapa-2- Google earth. Distancia entre las dos estancias de la familia Fros 
RELACult - Revista Latino-Americana de Estudos em Cultura e Sociedade

Revista Latinoamericana de Estudios en Cultura y Sociedad | Latin American Journal of Studies in Culture and Society V. 02, Ed. Especial, dezembro, 2016, p. 419-437| periodicos.claec.org e-ISSN 2016/Atual: 2525-7870 | e-ISSN 2015/2016: 2447-018X

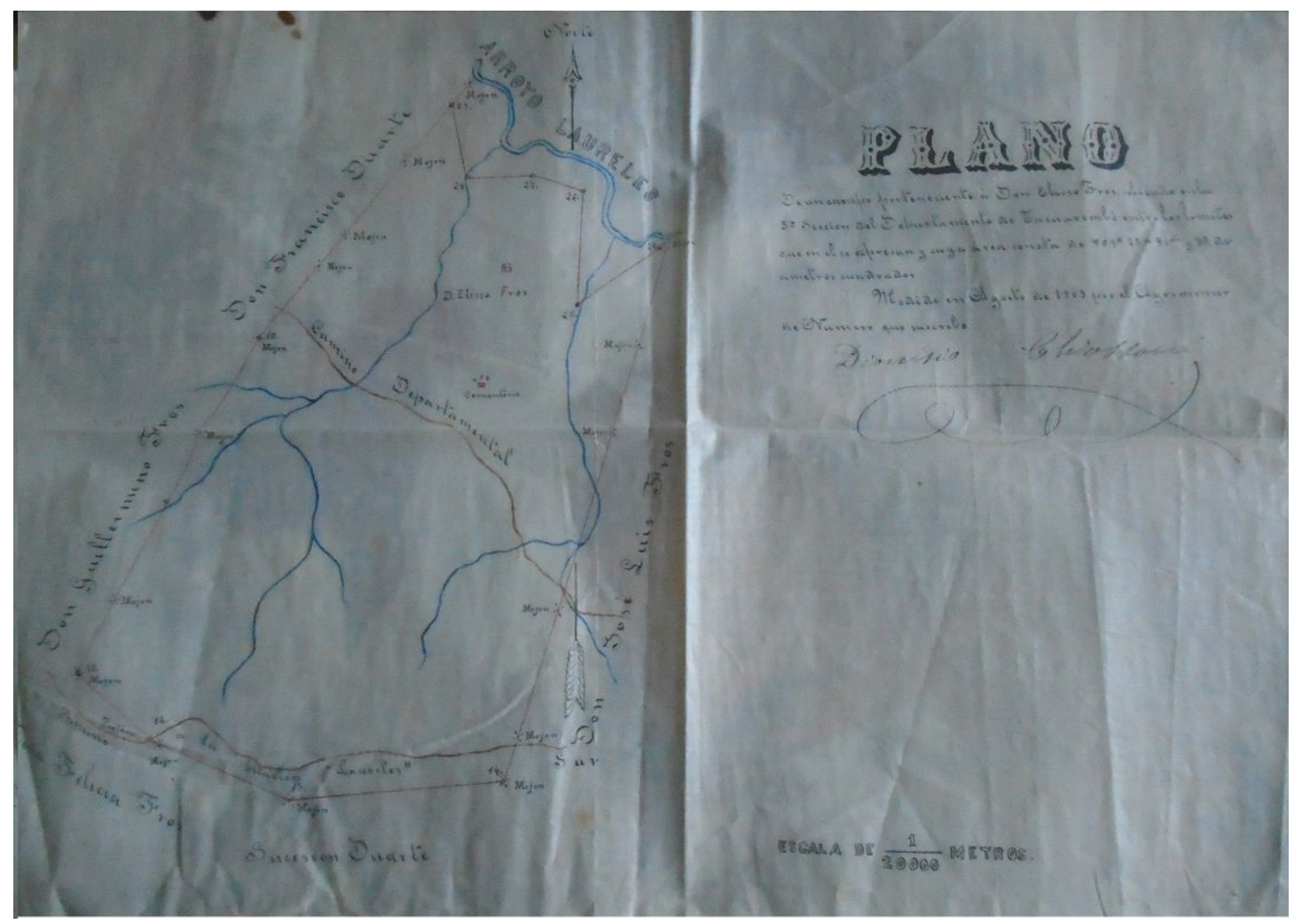

Foto- 3-Plano de la primera estancia de la propiedad Fros, brindada por D.F

Fuente: Archivo personal

Según los entrevistados con el apellido Fros la fotografía remite a la Primera Estancia construida en piedra. "Laureles allí, después por el año 1865, 1866 construyó donde hoy es el establecimiento y más tarde, exactamente en el año 1869 construyó la otra parte de la casa" (D.F, 08/2016).

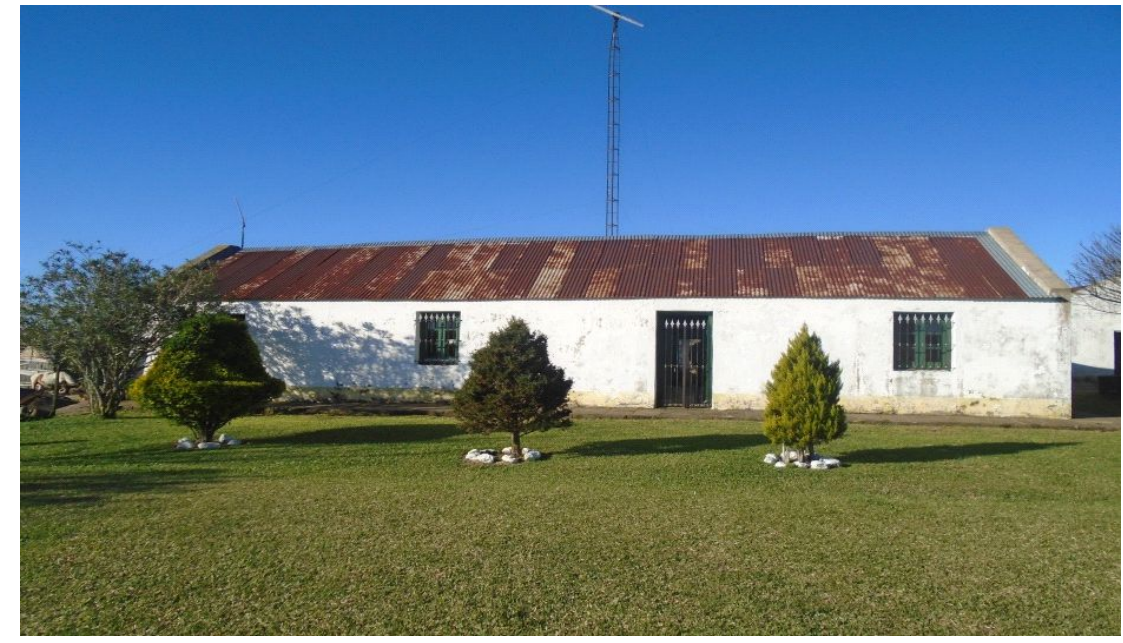

Foro- 4- Estancia de propiedad Fros en Laureles.

Fuente: Archivo personal. 
RELACult - Revista Latino-Americana de Estudos em Cultura e Sociedade

Revista Latinoamericana de Estudios en Cultura y Sociedad | Latin American Journal of Studies in Culture and Society V. 02, Ed. Especial, dezembro, 2016, p. 419-437| periodicos.claec.org e-ISSN 2016/Atual: 2525-7870 | e-ISSN 2015/2016: 2447-018X

"Don Toribio Alberto Fros, le da a la hija, nuestra abuela (...) Y lo que había era una casa de piedra la parte vieja. Sí, lo que queda ahora son los galpones del fondo, la pieza de esquila todo aquello" (T.R.S, 07/2016)

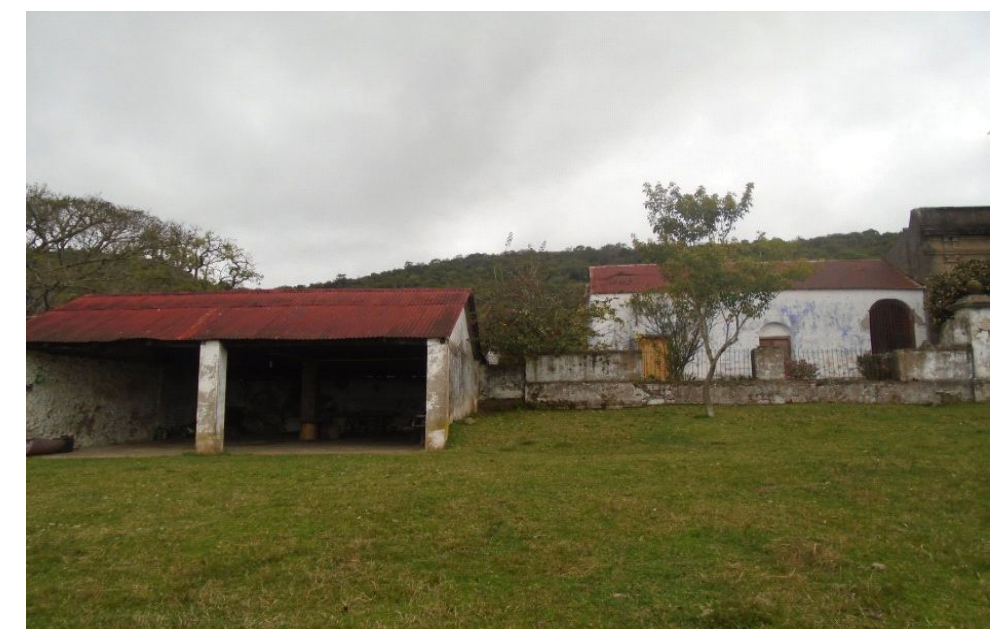

Foto- 5- Estancia de propiedad de los Fros en Bañado de Cañas.

Fuente: Archivo personal

Luego de observar la estructura y las fachadas de estos edificios se puede decir que se asemejan a la estructura de una estancia en Sao Leopoldo, la colonia alemana en Brasil, llamada "Feitoría Velha" (AURELIO PORTO, Pág: 81). Son construidas en piedra, las aberturas, tanto puertas como ventanas utilizan arco de medio punto por encima del dintel, su techo construido en teja. Con esto se puede apreciar que a pesar de los movimientos que realizaron en el territorio, siguen un mismo padrón de construcción.

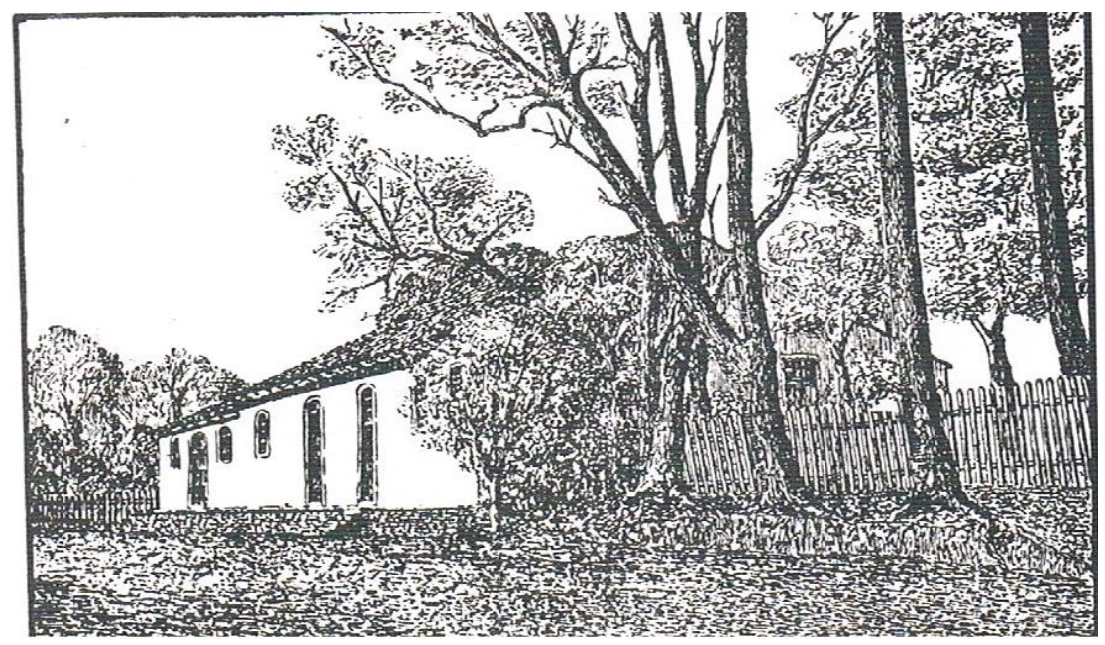

Imagen- 6- Feitoría Velha- São Leopoldo Brasil- Fuente: "O trabalho alemão no Rio Grande do Sul" Aurelio Porto; pág:81. 
Uno de los entrevistados, integrante de la familia Fros, la cual habitó en uno de sus establecimientos, aporta la distribución interna de las habitaciones según la jerarquía de la familia.

\begin{abstract}
Como toda estancia Colonial, establecimiento colonial, estaba la parte principal, la que daba al frente, la que se recibía las visitas, el comedor de las visitas, algún dormitorio de huésped o algo así y después (...)la casa de la familia, el matrimonio y las hijas mujeres, de este otro lado los hijos varones, por lo general cerraban un cuadro con un muro de piedra generalmente en la época, siempre había dormitorios allá , dormitorios acá y una casa principal, depende de la cantidad de hijos también, porque antes, a medida que iban quedando mayores los hijos ya se hacían sus dormitorios aparte. (Entrevista: T. R. S)
\end{abstract}

A partir de esta descripción se puede visualizar la descripción del lugar según una familia típicamente patriarcal, y cómo está distribuía estratégicamente para recibir bien a los invitados, y cuidar a las hijas mujeres, que en este tipo de familia serían sus reliquias. Describe como un lugar muy espacioso, esto a la vez también era estratégico ya que según los relatos de los entrevistados, realizaban muchas fiestas y conmemoraciones que siempre culminaban en bailes.

(...) y de repente bailes en casa de familias, los bailes familiares eso sí, eso era... era algo muy tradicional y fulano hacía en su casa y... iba todo el vecindario, a su vez el otro hacía para retribuirle lo que había hecho e iba todo el contingente para la casa de él, eso sí (...)" (Entrevista: D.F). "(...) familia muy alegre, muy divertida y muy unida, dice que, felicitaban, este, y que en esas visitas, visitas que iban y se quedaban en la casa, siempre terminaban en fiestas, viste, yerra, el nacimiento de alguien, un bautismo, o simplemente visita de cortesía de familia, (...) ya faenaban, una ternera o un cerdo y ahí ente todos elaboraban, las, las cosas ya sea chorizo, asado y cosas para ofrecerles a las visitas. Y sé que, terminada la, la faena y eso, cuando llegaban los vecinos se formaba baile, y más, mas, como te voy a decir, si había en la familia alguna muchacha en edad de casarse, entones, eran las, los, a fin de que se conocieran, armaban la fiestita de baile. Y fabricaban bombones, en, como es aquello, los bombones de huevos, como es, las yemitas (Entrevista: T.R.S).

\title{
3. Conclusión
}

Luego de recabar materiales y realizar el análisis pertinente de cada uno de ellos, como las fotos de las estancias, los bautismos recabados en la Curia de Tacuarembó, las entrevistas a diferentes personas con el apellido Fros y la bibliografía pertinente para dicha investigación, se puede afirmar que la familia Fros fueron migrantes de Alemania, llegaron a América, en territorios dominados por los portugueses, y luego se instalan en la región norte de la banda Oriental a partir de las segundas oleadas migratorias aproximadamente, fijándonos en el análisis de Patricia Demicherri (2013). La fecha recabada que más se aproxima a este hecho es la que está registrada en los documentos de la primera propiedad del 
territorio adquirido por Guillermo Alberto Fros, en la Banda Oriental "Título de Dn. Guillermo Alberto Fros" (Documento de propiedad; Brindado por D.F) documentado el 28 de enero del año 1856. “(...) tres oleadas migratorias de 1830 a 1843, de 1851 a 1870 , de 1880 a 1889."(PATRICIA DEMICHERRI, 2013, Pág 14). En estos períodos el continente americano, más precisamente las regiones de Brasil y La Banda Oriental eran los responsables de recibir a muchos de ellos. Por decisión de cada país, tanto Brasil como La Banda Oriental estaban dispuestos a recibirlo ya que necesitaban de su mano de obra y la situación en Europa no era de las mejores en esa época, claramente lo sostiene Partricia Demicherri en el libro "Familias Italianas en el Río de la Plata” “(...) tiempos difíciles en que sus habitantes comenzaron pensar en emigrar a América, (...) significaba su única salvación, la tierra prometida.”(PATRICIA DEMICHERRI, Pág 13).

La inmigración a nuestro territorio ha sido realmente muy significativa a que la cultura de nuestro país es una mezcla de muchas otras, puesto que las personas que habitan nuestras tierras son de regiones diferentes, son provenientes de lugares como Suiza, Rusia, África, Italia, Alemania, por lo tanto se puede decir que Uruguay en el siglo XIX fue producto de personas de otros lugares. Como bien ya se nombró en páginas anteriores, lo más importante de esta familia era investigar y conocer los aspectos más relevantes de su cultura, los elementos materiales e inmateriales que trajeron con ellos, qué costumbres adoptaron en el nuevo territorio y cuales conservaron de su cultura originaria alemana, que pueden ser visibles hasta hoy, ya que esas características hacen que se diferencien de las demás familias de migrantes establecidas en la región. "La identidad única de su propio pueblo, sin miedo a contradecirse" (KUPER, Pág 21), es decir que cada cultura tiene su propia identidad, que los hacen únicos.

Como toda familia inmigrante de otro territorio con su propia cultura llegan de forma homogénea "cada comunidad tenía su cultura propia con sus valores específicos que la separaba de todas las demás” (KUPER, Pág 35). Pero luego en el proceso de su establecimiento, se fue fusionando su cultura con la de la localidad "cruzar el atlántico...en busca de la esperanza... hasta soportar lentas y extenuantes colas en las instituciones oficiales, empuñando pasaportes y permisos de trabajo, es adquirir el hábito de vivir entre mundos, capturado en una frontera que atraviesa lengua, religión, música, vestimenta, apariencia y vida. Venir de otra parte, de "allá", no de "aquí", y encontrarse por lo tanto, de manera simultánea, "dentro" y "fuera" de la situación de que se trae, es vivir en las interacciones de historias y memorias (...)" (En ISABEL BARRETO, Pag: 18). Esta fusión se dio de tal manera que hoy a pesar de que se pueden identificar elementos que son propios 
de la cultura europea, las tradiciones de mantenerse la familia como unidad, sus festividades y algunos elementos materiales en cuanto a sus técnicas de construcción, y agrícolas, lo que más se identifica son elementos de la cultura Brasileña, cómo la lengua, sus costumbres la gastronomía, que se pudieron entrever en los análisis realizados de los materiales recabados.

Castillo (2012), afirma que para los seres humanos se torna fundamental relacionarse con sus pares, para así, orientar sus acciones e interactuar continuamente con la comunidad; de esta manera lograrán allegarse a lo que necesitan, aprender su comportamiento, normas y valores aceptados, así como también la cultura, costumbres, lenguaje, ideología, aparte de muchos otros aspectos que implica vivir en sociedad.

Para esto Bohannan (1992), plantea que los diferentes grupos sociales van a transitar por numerosos procesos de interacción, intensos y duraderos en el tiempo que van necesitar de variadas formas de comunicación, influyendo directamente en la modelación de cada individuo. En este proceso, la familia va a cumplir un papel fundamental al ser la cuna de la socialización primaria, en donde se gestan estos valores y principios formadores de la conducta. En cuanto a los aspectos específicamente inmateriales obtenidos como algunas descripciones, se los identifica como una familia unida, con hábitos de trabajo , tranquila, comparándola a simple vista con otras familias de la región, ésta es muy tradicional, sus propiedades han sido heredadas de generación en generación por hijos varones con el mismo apellido, la misma sangre.

(...)este aquí es un dato importante que siempre perteneció a la misma familia, desde que él compró, cuando murió Guillermo Alberto, quedo Florentino, cuando murió Florentino, Eliceo, el padre de papá, cuando murió Eliceo quedó papá, murió papá quede yo(...) (Entrevista: D.F.)

Se cree pertinente sumarle a esta descripción de la familia la visión eurocéntrica, es una forma de relación históricamente lineal, unidireccional, están inmersos un patrón de conducta invariable. El eurocentrismo propone al modelo occidental como el mejor, con respecto a los demás, es decir, "propone a toda la imitación del modelo occidental como única solución a los desafíos de nuestro tiempo." (SAMIR AMIR, pág.9) Para ese trabajo es conveniente abordar el eurocentrismo en el campo cultural e ideológico.

"La cultura evidencia lo que ese grupo es. La identidad nos permite decir, hablar, construir un discurso sobre lo que pensamos que somos." (PATRICIO GUERRERO ARIAS, pág. 104). La cultura permite que el hombre no solo se adapte a su entorno sino que haga que este se adapte a él." (DENYS CUCHE, 1966, pág. 7). Con esto, se percibe la importante 
influencia que este fenómeno llamado eurocentrismo, tiene sobre la identidad de los grupos sociales. Esto se percibe en la forma en que dichos grupos se van a relacionar con otros y como se organizan al momento de asentarse.

\section{Bibliografía:}

ARRIAGA Rodrigues Juan Carlos; "el concepto de frontera en la perpectiva humana" 2012.

AROCENA Felipe; "La contribución de los inmigrantes en Uruguay"; 2007.

ALDAIR Marli Lando,y CRUXEN Barros Eliane; "A colonização alemã no Rio do Grande do Sul” Uma interpretação sociológica. Porto Alegre; Movimento/Instituto Estadual do Livro, 1976.

BOHANNAN, Paul. 1992 "Para Raros, Nosotros" Introducción a la antropología cultural. Madrid, España. Ediciones AKAL, S.A.BARRETO Messano Isabel; "Canarios de ayer La inmigración canaria en el Uruguay".

BERGERON Luis, y otros; "Historia Universal sigo XXI, Volumen 26, La Época de las revoluciones europeas 1780-1848”.

CASTILLO Romero, Jorge Rodrigo. "Sociología de la Educación”; Primera Edición 2012.

CUCHE Denys ;"La noción de cultura en las ciencias Sociales” Ediciones nueva visión SAIC; 1999; Argentina.

DEMICHERRI Patricia; "Familias Italianas en el Rio de la Plata", Montevideo, Linardi y Risso 2013.

DÍAZ Aja Antonio; “TEMAS EN TORNO A UN DEBATE SOBRE LAS MIGRACIONES INTERNACIONALES”, La Habana, Cuba, 2004.

FALICOV, Estela y LIFSZYC Sara ;(2002), Sociología. Buenos Aires, Aique. Página 187.

FERNÁNDEZ Kara; “Liberalismo y nacionalismo en la Europa del siglo XIX”, 2010.

GUERRERO Arias Patricio, "LA CULTURA estrategias conceptuales para entender la identidad, la diversidad, la alteridad y la diferencia.", Abya-Yala, 2002.

GIDDENS Anthony ;(1999), Sociología. Madrid, Alianza.

KONETZKE Richard; "Historia Universal del siglo XXI, América Latina, La época colonial" México, 2010.

KUPER Adam “Cultura: la visón de los antropólogos”, A \& M grafic. S.L, Barcelona 2001.

MARRERO Adriana; "Introducción a la Sociología”. Editorial MASTERGRAFT Sal. Medo. Octava edición 2006; Uruguay. 
PORTO Aurelio; "O trabalho alemão no Rio Grande do Sul"

QUIJANO Anibal; "Colonialidad del poder y clasificación social" Buenos Aires, 2014.

QUIJANO Anibal; “Colonialidad del Poder y Des/Colonialidad del Poder”.

REYES Johan, "Memoria individual y memoria colectiva: Paùl Ricoeur” Venezuela, 2008. RIVERO de Arhancet, Mabel. Ramos Cabanellas, Beatriz. "Nuevo régimen legal del matrimonio. Matrimonio Igualitario”. 2014 Segunda edición. Fundación de Cultura Universitaria. Uruguay.

Prof. SAMANIEGO Mrcedes, "Historia Política y Social Contemporánea. S- XIX", UNIVERSIDAD DE SALAMANCA FACULTAD DEDERECHO ESPECIALIDAD: CIENCIAS POLITICAS Y DE LA ADMINISTRACION, 2008-2009.

SAMIR Amin;"EL EUROCENTRISMO critica de una ideología", siglo veintiuno editores, 1989.

TAYLOR, Edward 1871; VILLEGAS Juan S. J. "Historia de la evangelización en el Uruguay al Norte del Río Negro".

\section{Fuentes:}

Entrevista a D.F

Entrevista a A.F

Entrevista a T.R.S 